\title{
A basic study of the effect of the shielding method with polyglycolic acid fabric and fibrin glue after endoscopic submucosal dissection
}

Authors

Institutions
Tsujimoto Hiroyuki ${ }^{1}$, Yamanaka Kohki ${ }^{1}$, Miyamoto Hiroe ${ }^{1}$, Horii Tsunehito ${ }^{1}$, Abe Rie ${ }^{1}$, Tanaka Shota', Torii Hiroko ${ }^{1}$, Ozamoto Yuki', Toshitaka Takagi ${ }^{1}$, Takimoto Kengo ${ }^{2}$, Torii Takashi ${ }^{3}, K^{2}$ Konishi Hideyuki ${ }^{3}$, Takamori Hideki ${ }^{4}$, Hagiwara Akeo ${ }^{1}$

Institutions are listed at the end of article. submitted 9. March 2016 accepted after revision 13. September 2016

\section{Bibliography}

Dol http://dx.doi.org/

10.1055/s-0042-118208

Endoscopy International Open

2016; 04: E1298-E1304

(c) Georg Thieme Verlag KG

Stuttgart · New York

E-ISSN 2196-9736

\section{Corresponding author}

Hiroyuki Tsujimoto, MD, PhD

Division of Medical Life System

Department of Life

and Medical Science

Doshisha University

1-3 Tatara-Miyakodani

Kyotanabe, Kyoto 610-0321

Japan

Fax: +81-774-65-6887

sk108621@mail.doshisha.ac.jp

\section{License terms}

\section{(9)(1) $\ominus \circledast$}

Background and study aims: Recently, the shielding method with polyglycolic acid (PGA) fabric and fibrin glue (P-F method) has been reported to prevent serious complications after endoscopic submucosal dissection (ESD). However, the effectiveness and mechanism to prevent complications by this method remain unclear and the corresponding basic research has not been fully conducted.

Methods and results: We examined the effectiveness and mechanism of the P-F method, using a surgical ESD model of canine stomach and in vitro experiments. In the model experiment treated by P-F method or no treatment (control), ulcer perforation or penetration occurred only in the control group, but not in the P-F group. Microscopically, the P-F group showed less damages of the ulcer lesion than that of the control group,

\section{Introduction}

$\nabla$

The use of ESD has become widespread as an effective treatment for early-stage gastrointestinal tumors [1-4]. ESD can remove a whole circumference mucosa around tumors en bloc with minimal invasiveness against surgical operations [1, 3]. It can also resect larger areas with tumors compared with endoscopic mucosal resection (EMR), and reduce the risk of local recurrence by accurate histopathologic diagnosis $[2,4]$. However, ESD occasionally involves serious complications such as postoperative perforation or bleeding $[1,3]$. Therefore, methods for preventing those complications are required.

Previously, a method to close mucosal defects after ESD with clips (clipping method) was reported [5]. However, this method involved technical difficulties in closing large mucosal defects and the duration of clip persistence has not been clarified $[6,7]$. Recently, Takimoto et al. reported a method of shielding the mucosal defects after ESD with PGA fabric and fibrin glue (P-F method) to showing thicker granulation tissues including PGA fibers on the third day and excellent mucosal regeneration on the fourteenth day. In vitro culture experiments showed that fibroblasts proliferated at a significantly higher rate on PGA than on fibrin or a complex thereof. However, under hydrochloric acid treatment, fibroblasts were protected by fibrin, followed by the complex of both, and PGA.

Conclusion: The P-F method exhibited a protective effect against gastric juice by fibrin glue to reduce tissue damages and a scaffold function of PGA fabric to induce better granulation formation at the earlier phase, resulting in excellent longterm tissue repair, on ulcer lesion following ESD, although the results were based on basic experiments.

prevent related complications [6-8]. However, the effectiveness and mechanism by which this methods prevents complications remain unclear and the corresponding basic research has not been fully conducted [9]. In the current study, we attempted to confirm the effectiveness of the P-F method using a surgical model similar to ESD in the canine stomach. In addition, to examine its mechanism, we investigated the role of each material of the P-F method using in vitro culture experiments.

\section{Materials and methods \\ $\nabla$}

Surgical ESD model experiments in canine stomach

Twenty female dogs aged approximately 10 months and weight $9.4 \pm 0.3$ (mean \pm SD) were purchased from Shimizu Laboratory Animal Supply Co. (Kyoto, Japan). All animal care, housing, and surgical procedures were in accordance with the guide for the care and use of laboratory ani- 


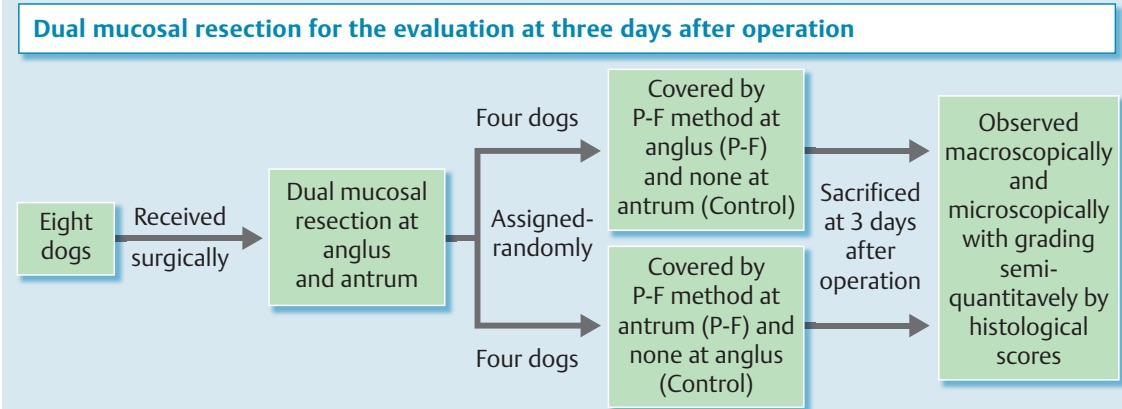

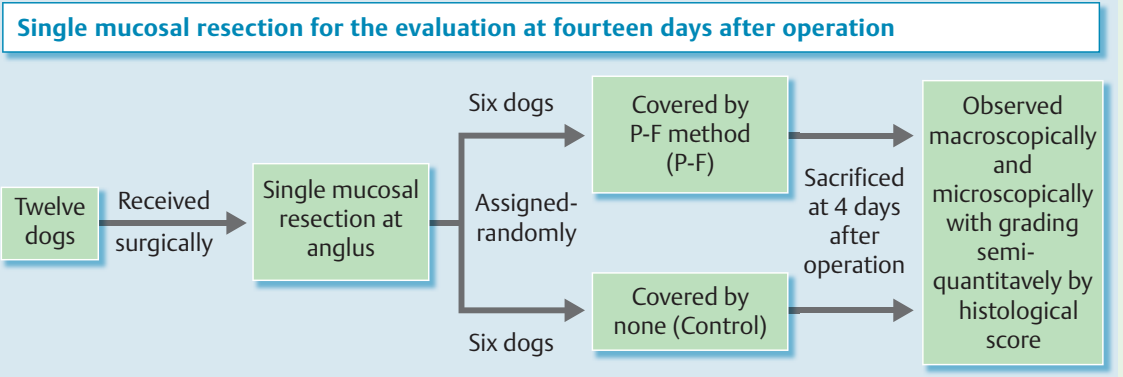
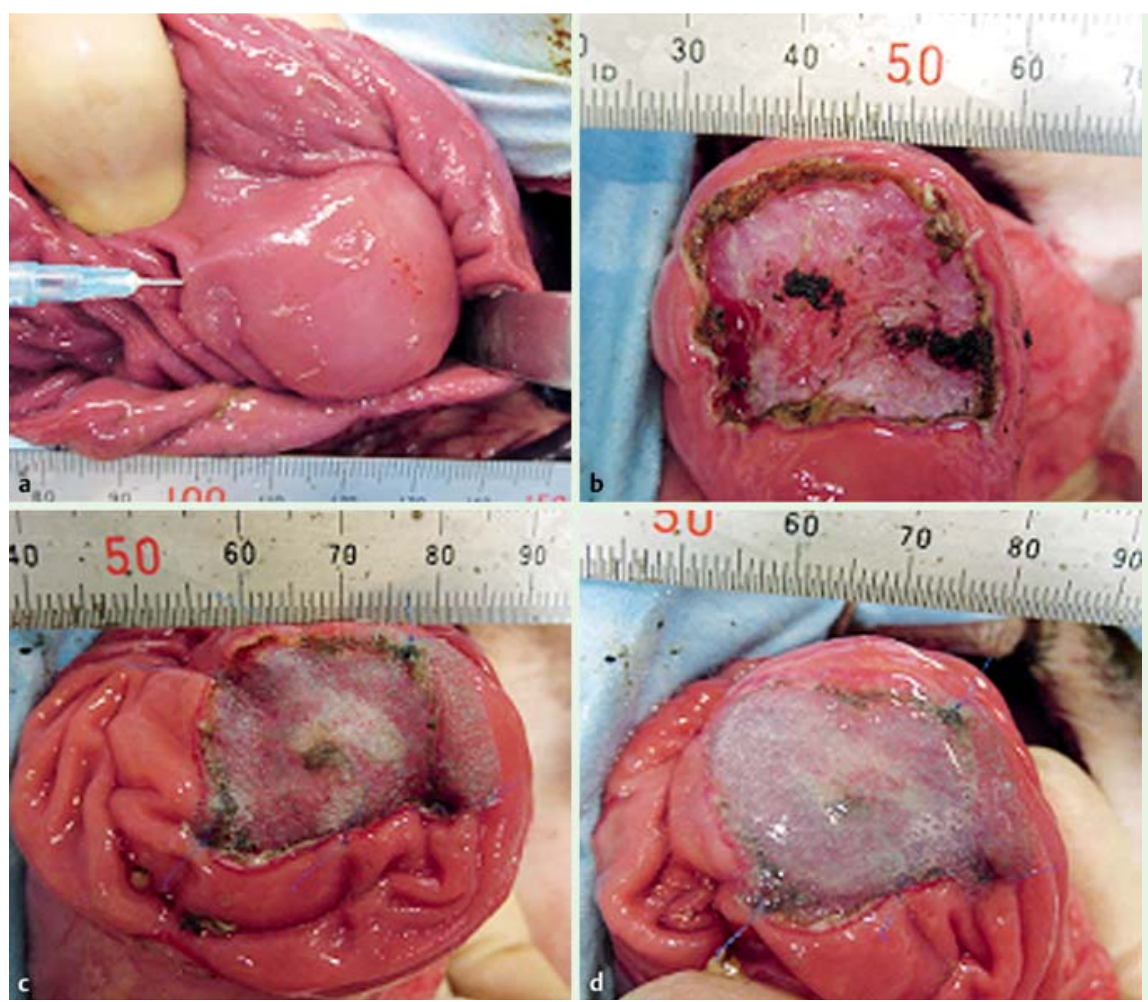

Fig. 2 Procedures for the surgical model using the canine stomach. a Injection of saline into the submucosal layer on the posterior wall of stomach. b After removal of the elevated mucosa with an electric knife. c After patching the PGA sheet and fixing with nylon sutures. $\mathbf{d}$ After spraying fibrin glue on the patched PGA sheet.

Fig. 1 Flow diagram of all animal experiments.

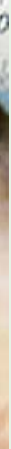

mals published by the National Academy of Sciences and the institutional guidelines of the Committee for Animal Research of Doshisha University. The procedures used in the animal experiments are summarized in $\bullet$ Fig. 1.

Under venous administration of pentobarbital (Somnopentyl ${ }^{\circledR}$, Kyorituseiyaku Co., Tokyo, Japan) at a dose of $0.5 \mathrm{~mL} / \mathrm{kg}$, all dogs received a surgical incision on the anterior wall of the lower stomach and $6 \mathrm{~mL}$ of saline was injected into the submucosal layer on the posterior wall of the angler region by injector $(10 \mathrm{~mL}$ with a $25-G$ needle) ( Fig. 2 a). Then, the elevated mucosa was removed (a rectangle measuring approximately $3 \mathrm{~cm} \times 2 \mathrm{~cm}$ in size) with an electric knife (Hyfrecator $2000^{\circledR}$, ConMed, Utica,
NY, USA; setting at Cut: 50 and Coagulation: 50) ( $\bullet$ Fig. 2 b). For 8 of the dogs, an additional mucosal resection measuring the same size was done at the antral region with a 4-cm distance between the 2 lesions. Dogs with dual resection were randomized in double-blind fashion to treatment of one of the regions with a PGA sheet, as were half the dogs that had undergone single resection. For the procedure, a $3 \mathrm{~cm} \times 2 \mathrm{~cm}$ PGA sheet (Neoveil ${ }^{\circledR}$, GUNZE Co., Tokyo, Japan) was patched and fixed with 7-0 nylon sutures ( $\bullet$ Fig. 2c). Then, $1 \mathrm{~mL}$ each of solution A (fibrinogen) and solution $B$ (thrombin) of a fibrin glue kit (Beriplast ${ }^{\circledR}$ P Combi-Set, CSL Behring LLC, King of Prussia, PA, USA) was simultaneously sprayed on the PGA sheet (P-F group) ( $\bullet$ Fig. 2d). Finally, 4 of 8 
Table 1 Histologic scores for the depth of ulcer, granulation tissue formation, connective tissue formation and mucosal regeneration of ulcer lesions

\begin{tabular}{l|l}
\hline $\begin{array}{l}\text { Category and description } \\
\text { (Depth of ulcer or ulcer scar) }\end{array}$ & Score \\
\hline $\begin{array}{l}\text { Within the submucosal layer } \\
\text { Up to the muscular layer }\end{array}$ & 1 \\
\hline $\begin{array}{l}\text { Beyond the muscular layer, including perforation } \\
\text { or penetration }\end{array}$ & 2 \\
\hline \begin{tabular}{l} 
(Granulation tissue formation at the bottom of an ulcer lesion ) \\
\hline $\begin{array}{l}\text { Thinner layer of granulation tissue than the thickness } \\
\text { of the normal submucosal layer }\end{array}$
\end{tabular} & 0 \\
\hline $\begin{array}{l}\text { Layer of granulation tissue nearly equal to the thickness } \\
\text { of the normal submucosal layer }\end{array}$ & 1 \\
\hline $\begin{array}{l}\text { Thicker layer of granulation tissue than the thickness } \\
\text { of the normal submucosal layer }\end{array}$ & 2 \\
\hline \begin{tabular}{l} 
(Connective tissue formation at the bottom of an ulcer lesion) \\
\hline Sparse or focal connective tissue formation
\end{tabular} & 0 \\
\hline \begin{tabular}{l} 
Diffuse connective tissue formation \\
\hline Thick and dense connective tissue formation
\end{tabular} & 1 \\
\hline \begin{tabular}{l} 
(Regeneration of the mucosal layer at an ulcer lesion) \\
\hline No regeneration of the mucosal layer
\end{tabular} & 2 \\
\hline $\begin{array}{l}\text { Sparse or focal regeneration of the mucosal layer } \\
\text { Full regeneration of the mucosal layer }\end{array}$ & 0 \\
\hline
\end{tabular}

dogs received shielding by P-F method at anglus regions and the remaining dogs received shielding at antral regions. Then, 6 of 12 dogs with single resection received the P-F method. For the remaining lesions in the dogs with dual or single resection, none of the lesions were covered (control group).

After closure of the abdominal walls, each dog received prophylactic antibiotics (Penicillin G Potassium, 10,000 units/kg, Meiji Seika Ltd., Tokyo, Japan) subcutaneously for 3 days after surgery. However, no antiulcer agent was administered so that only the shielding effect of P-F method could be evaluated. All dogs were monitored carefully and evaluated on Day 3 or Day 14 after the operation.

Three days after the operation, all 8 dogs with dual resection were sacrificed and their wounds were observed macroscopically and microscopically semi-quantitatively graded according to histologic scores ( Table 1 ). Fourteen days after the operation, the remaining 12 dogs with single resection were sacrificed and observed in the same manner as those at the 3-day time point. Statistical comparisons were made by using the Mann-Whitney's U test. A P value less than 0.05 was considered to be significant.

\section{In vitro culturing experiments}

Evaluation of fibroblast proliferation on each material in the P-F method as a scaffold

To evaluate the potential of each material as a scaffold, we examined fibroblast proliferation on fibrin, PGA, or a complex thereof as follows ( $\bullet$ Fig. 3 a). In the fibrin group, we sprayed $0.3 \mathrm{~mL}$ each of solutions $A$ and $B$ in a fibrin glue kit (Veriplast ${ }^{\circledR}$ ) per well of a 24-well culture plate. In the PGA group, we placed the PGA fabric $\left(\right.$ Neoveil $^{\circledR}$ ) in the bottom of each well. In the P-F group, we placed PGA fabric onto each well, and sprayed $0.3 \mathrm{~mL}$ each of solutions A and $\mathrm{B}$ together per well. After $10^{4}$ rat fibroblasts with culture medium were poured onto the wells with or without each material, the viable cell number in each well was counted using the ATPLite ${ }^{\circledR}$ kit (Perkin-Elmer Inc., Waltham, MA, USA) at 1, 3, 5 and 7 days after seeding and culturing ( $n=4$ for each group). Statistical comparisons at each time point were made using the New-

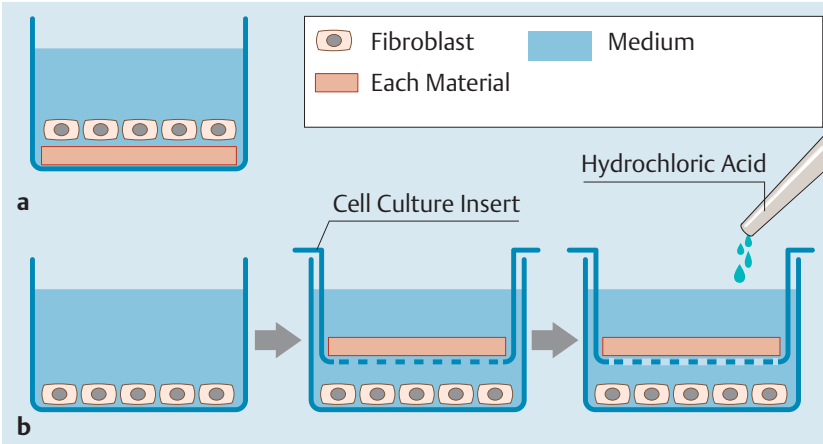

Fig. 3 Schema of in vitro experiments. a The schema of the experiment to evaluate fibroblast proliferation on each material in the P-F method as a scaffold. $\mathbf{b}$ The schema of the experiment to evaluate protective effect of each material in the P-F method on fibroblasts cultured under hydrochloric acid treatment.

man-Keuls test following ANOVA (analysis of variance). A P value less than 0.05 was considered to be significant.

Evaluation of the protective effect of each material in the P-F method on fibroblasts cultured under hydrochloric acid treatment

To evaluate the protective effect of each material against hydrochloric acid, $10^{4}$ rat fibroblasts were cultured onto each well of a 24-well cell culturing plate for 12 hours. We applied $0.05 \mathrm{~mL}$ each of solutions A and B of a fibrin glue kit (Veriplast $\left.{ }^{\circledR}\right)$ in the fibrin group, PGA fabric $\left(\right.$ Neoveil $\left.^{\circledR}\right)$ in the PGA group, PGA fabric with $0.05 \mathrm{~mL}$ each of solutions A and B in the P-F group, and nothing in the control group into each cell culture insert ${ }^{\circledR}$ (Falcon, Flanklin Lakes, NJ, USA), respectively. After setting the inserts with or without each materials on the wells with fibroblasts, we added $21 \mu \mathrm{L}$ of $2 \mathrm{M}$ hydrochloric acid and $500 \mu \mathrm{L}$ of the culture medium onto the inserts. (approximate $\mathrm{pH} 4.5$ at 3 hours after mixing in our preliminary study) ( $\bullet$ Fig.3b). After culturing, the number of viable cells in each well was counted using the ATPLite ${ }^{\circledR}$ kit at $0,1,3,6,12,24$, and 72 hours ( $n=4$ for each group). Statistical comparisons were made using the non-paired Student's $t$-test between each material and control at each time point. A $P$ value less than 0.05 was considered to be significant.

\section{Results \\ $\nabla$}

\section{Surgical ESD model experiments in canine stomach}

Three days after the operation, all 8 dogs with dual resection were sacrificed and observed macroscopically and microscopically semi-quantitatively graded according to histologic scores ( $\bullet$ Table 1). Although there was no mortality associated with the surgical procedures, complete perforation of the gastric wall was noted in 3 dogs in the control group ( 2 at the anglus and 1 at the antrum) ( $\bullet$ Fig.4a). However, no such severe complication was seen in the lesions covered with the P-F method. Inside the stomach, the PGA fabrics still remained covering the ulcer lesions, but no remaining fibrin clots was not seen ( $\bullet$ Fig.4c). Microscopically, although most of the remaining PGA fabric was removed during specimen processing, the depth of the ulcer lesions in the P-F group was less than that in the control group with significantly lower scores. In addition, more formation of granulation tissue at the bottom of the ulcer lesions was noted in the P-F group than in the control group ( $\bullet$ Fig. 4 b, $\bullet$ Fig. 4 d, $\bullet$ Table 2 ). Inter- 


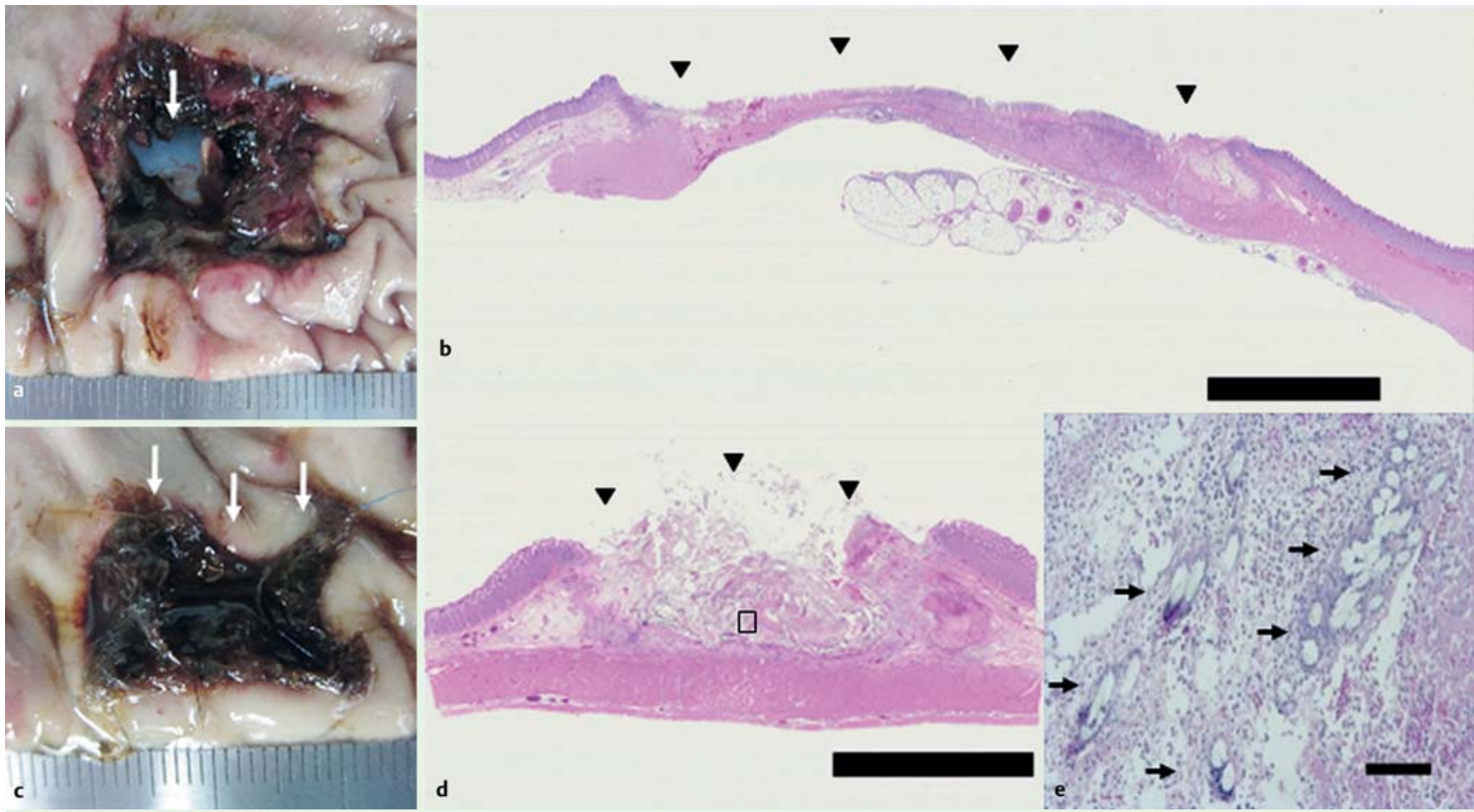

Fig. 4 Macroscopic and microscopic views of the ulcer lesions on the third day. a Macroscopic view of the ulcer lesion in the control group. The white arrow indicates complete perforation of the large ulcer lesion. b Microscopic view of the ulcer lesion in the control group. Black arrowheads indicate a deep ulcer lesion involving a severely denatured muscular layer and poor granulation tissues in the submucosal layer. Black bar indicates $10 \mathrm{~mm}$. c Macroscopic view of the ulcer lesion in the P-F group. The white arrow indicates the ulcer lesion covered with PGA fabric. $\mathbf{d}$ Microscopic view of the of the ulcer lesion in the P-F group. Black arrowheads indicate the ulcer lesion with an almost intact muscular layer and dense granulation tissues in the submucosal layer. Black bar indicates $10 \mathrm{~mm}$. e Magnified view of the black square in d. Black arrows indicate fibers of PGA fabric surrounded by granulation tissues in the submucosal layer. Black bar indicates $50 \mu \mathrm{m}$.

estingly, some fibers from the PGA fabric were observed among the granulation tissues but no fibrin glue ( $\odot$ Fig. $4 \mathrm{e}$ ).

Fourteen days after the operation, the remaining 12 dogs ( 6 in each group) with single resection were sacrificed and observed in the same manner as the others at the 3-day time point. Although there were no mortalities, penetration of the ulcer lesions into the adjacent pancreas was observed in 2 dogs in the control group, but none in the P-F group. The PGA fabric remained covering the ulcer lesions in 5 of 6 ulcer lesions in the P-F group ( $\odot$ Fig. 5 a, $\odot$ Fig. 5 c).

Microscopically, the depth of the ulcer lesions and the connective tissue formation at the bottom of ulcer lesions in the P-F group tended to be less and more than those of the control group, respectively. Interestingly, the P-F group showed excellent mucosal regeneration at the bottom of the ulcer lesions $(\bullet$ Fig. 5 b, $\odot$ Fig. $5 \mathrm{~d}$, $\odot$ Table 2). A small amount of fiber from the PGA fabric was also detected under the regenerated mucosal layer ( $\bullet$ Fig. $5 \mathbf{e})$.

\section{In vitro culturing experiments}

Evaluation of fibroblast proliferation on each material in the P-F method as a scaffold

As shown in $\odot$ Fig. 6 a, the number of fibroblasts in the PGA group was significantly higher than those in the fibrin and P-F groups at all time points and those in the control group at Day 5 and Day 7 . There was no significant difference between the fibrin and P-F groups at all time points.
Evaluation of the protective effect of each material in the P-F method on fibroblasts cultured under hydrochloric acid treatment

As shown in 0 Fig. $\mathbf{6 b}$, the number of fibroblasts in the fibrin group were significantly higher than those in the PGA and control groups at all time points except 6 hours and those in the P-F group at 24 hours and 72 hours. The number of fibroblasts in the P-F group were significantly higher than those in the PGA and control groups at $1,3,12$, and 24 hours. There was no significant difference between the PGA and control groups at any time point.

\section{Discussion}

Before the use in the field of gastrointestinal endoscopy, the P-F method was used commonly in the field of surgery to prevent air, bile, and pancreatic juice after pneumonectomy [10], hepatic resection [11], and pancreatectomy [12], respectively. In addition, Shinya et al. reported that the P-F method provided the strongest adhesive force and sealing effect, compared with fibrin glue alone or other biomaterials in combination with fibrin glue [13]. On the basis of that background, in this study, we employed a surgical ESD model and P-F method with sutured PGA fabric. Because the sutured PGA fabrics remain for a long time, it is more convenient to evaluate the effect of P-F method. In addition, no antiulcer agent was administered so that only the shielding effect of the P-F method could be evaluated, because an antiulcer agent might mask or attenuate the difference between the P-F method and control. In the experiment using the model, serious 
Table 2 Summary of histologic scores for the depth of ulcer, granulation tissue formation, connective tissue formation and mucosal regeneration of ulcer Lesions

\begin{tabular}{|c|c|c|c|c|}
\hline & \multicolumn{2}{|l|}{3 days $(n=8)$} & \multicolumn{2}{|c|}{14 days $(n=6)$} \\
\hline \multicolumn{5}{|c|}{ (Depth of ulcer or ulcer scar) } \\
\hline P-F method & $0.38 \pm 0.52$ & $P<0.05$ & $0.33 \pm 0.52$ & \multirow{2}{*}{$\begin{array}{l}\text { n.s. } \\
(P=0.057)\end{array}$} \\
\hline Control & $1.25 \pm 0.71$ & $(P=0.020)$ & $1.17 \pm 0.75$ & \\
\hline \multicolumn{5}{|c|}{ (Granulation tissue formation at the bottom of an ulcer lesion ) } \\
\hline P-F method & $1.38 \pm 0.74$ & $P<0.05$ & $1.17 \pm 0.75$ & \multirow{2}{*}{$\begin{array}{l}\text { n. s. } \\
(P=0.206)\end{array}$} \\
\hline Control & $0.50 \pm 0.53$ & $(P=0.024)$ & $0.67 \pm 0.52$ & \\
\hline \multicolumn{5}{|c|}{ (Connective tissue formation at the bottom of an ulcer lesion) } \\
\hline P-F method & $0.50 \pm 0.53$ & n.s. & $1.33 \pm 0.52$ & \multirow{2}{*}{$\begin{array}{l}\text { n. s. } \\
(P=0.056)\end{array}$} \\
\hline Control & $0.13 \pm 0.35$ & $(P=0.117)$ & $0.67 \pm 0.52$ & \\
\hline \multicolumn{5}{|c|}{ (Regeneration of the mucosal layer at an ulcer lesion) } \\
\hline P-F method & $0.00 \pm 0.00$ & \multirow{2}{*}{ no $P$ value } & $1.33 \pm 0.52$ & \multirow{2}{*}{$\begin{array}{l}P<0.05 \\
(P=0.030)\end{array}$} \\
\hline Control & $0.00 \pm 0.00$ & & $0.50 \pm 0.55$ & \\
\hline
\end{tabular}

Data are presented as the means \pm SD.

Statistical comparisons were made by the Mann-Whitney's $U$ test.

A $P$ value less than 0.05 was considered to be significant.

n. s., not significant.
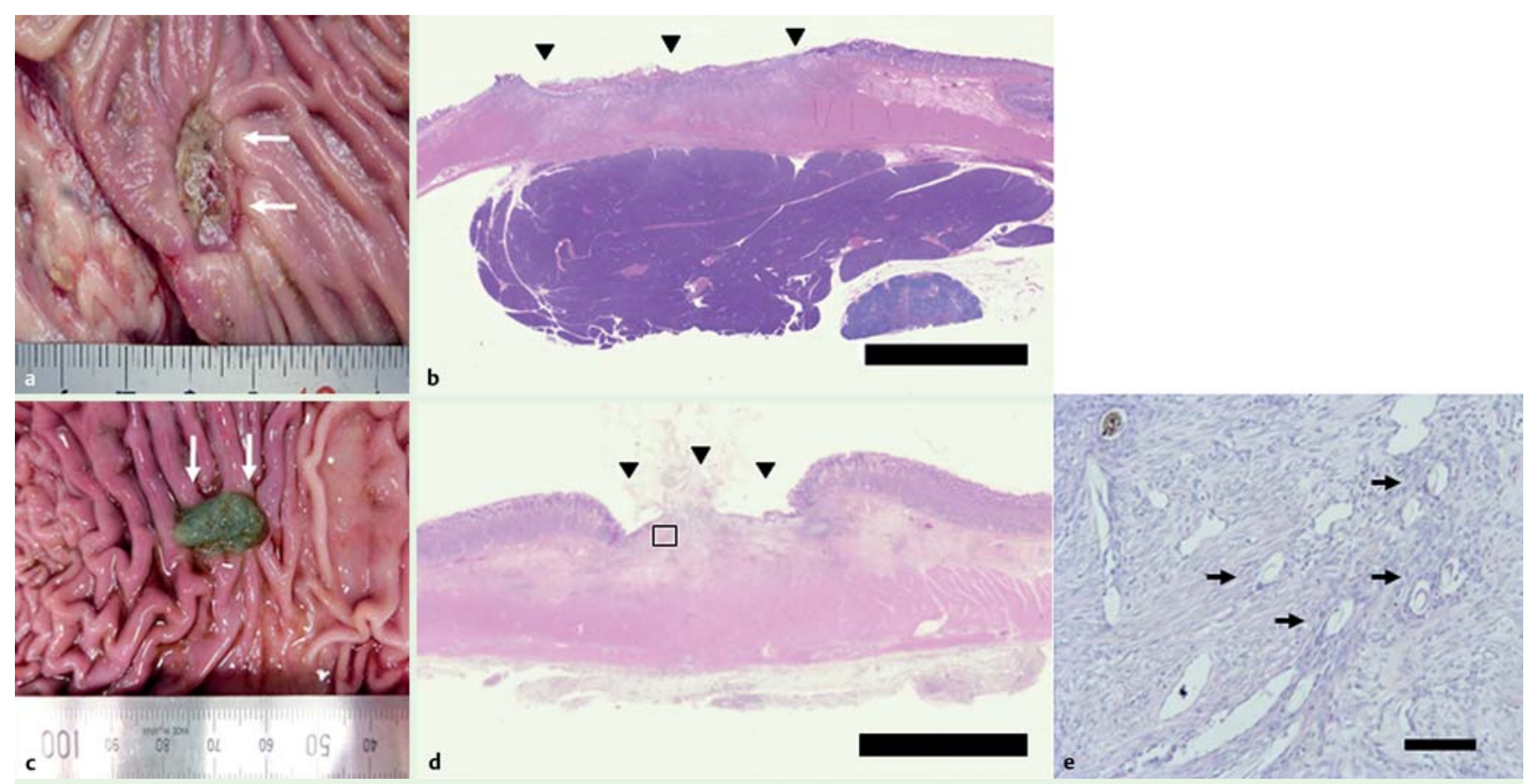

Fig. 5 Macroscopic and microscopic views of the ulcer lesions on Day 14. a Macroscopic view of the ulcer lesion in the control group. The white arrow indicates the ulcer lesion. $\mathbf{b}$ Microscopic view of the ulcer lesion in the control group. Black arrowheads indicate the ulcer lesion penetrating into the adjacent pancreas with a thin denatured muscular layer and a poorly regenerated mucosal layer. Black bar indicates $10 \mathrm{~mm}$. c Macroscopic view of the ulcer lesion in the P-F group. The white arrow indicates the ulcer lesion covered with PGA fabrics. $\mathbf{d}$ Microscopic view of the of the ulcer lesion in the P-F group. Black arrowheads indicate the ulcer lesion with an almost intact muscular layer and a well-regenerated mucosal layer. Black bar indicates $10 \mathrm{~mm}$. e Magnified view of the black square in $\mathbf{d}$. Black arrows indicate fibers of PGA fabric in the regenerated submucosal layer. Black bar indicates $50 \mu \mathrm{m}$.

complications such as perforation of the gastric wall or penetration to the adjacent pancreas was only observed in the control group, but not in the P-F group. That result indicates that the P-F method can prevent such postoperative complications.

Histologically, the depth of the ulcer lesions in the P-F group was less than that in the control group. In addition, in the ulcer lesions of the P-F group, thicker granulation tissues on the Day 3 and more abundant connective tissues and mucosal regeneration on the Day 14 were noted compared with that in the control group. These findings suggest that the P-F method can effectively cover the ulcer lesion and prevent damage to the ulcer region from gastric juice at the earlier phase and also contribute to excellent long-term tissue repair and mucosal regeneration. Interestingly, remnant PGA fibers were found in the granulation tissue at the bottom of the ulcer lesions in the P-F group. This suggests that the PGA fiber may act partially as a scaffold for inflammatory cells and fibroblasts to form the thicker granulation layers.

Based on these results, we subsequently conducted 2 in vitro experiments to investigate the roles of each material in the P-F method. The experiment to evaluate fibroblast proliferation on each material showed that fibroblasts on PGA alone proliferated at a significantly higher rate than those on fibrin alone or a complex of PGA and fibrin, indicating that PGA acts as scaffold for fibroblast proliferation. We then evaluated the protective effect 


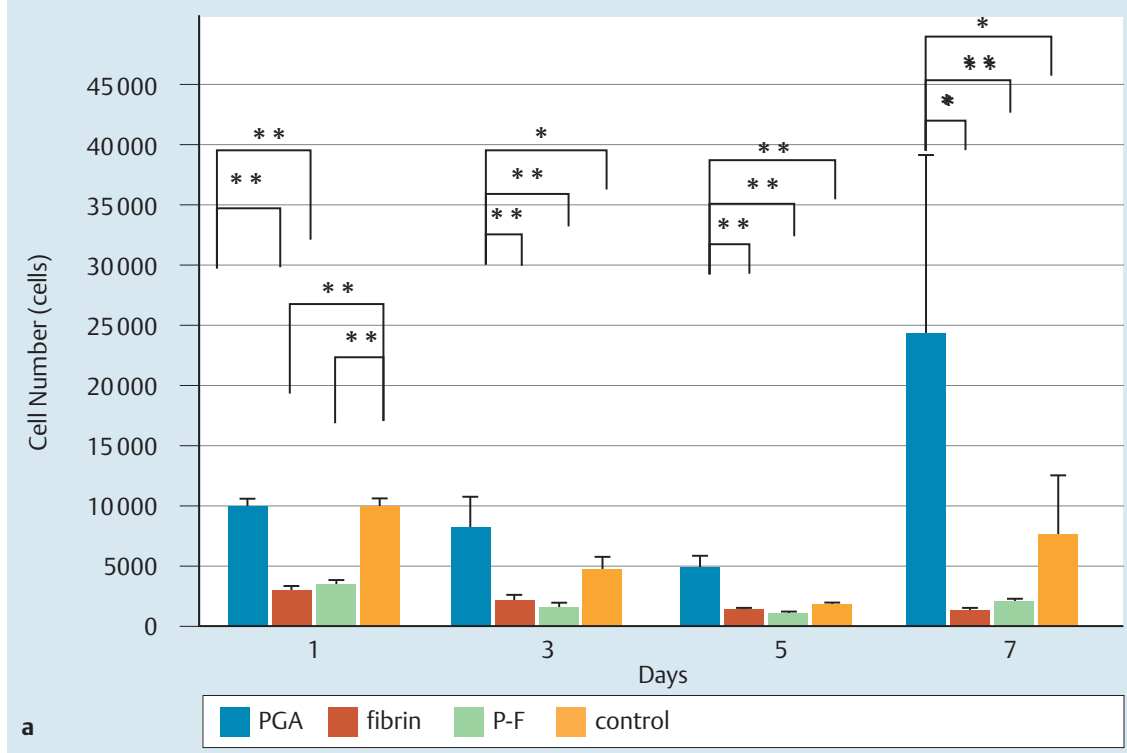

Fig. 6 Results of in vitro experiments. a The result of experiment to evaluate fibroblast proliferation on each material in the P-F method as a scaffold. ${ }^{*}: P<0.05,{ }^{* *}: P<0.01$. $\mathbf{b}$ The result of experiment to evaluate protective effect of each material in the P-F method on fibroblasts cultured under hydrochloric acid treatment. * $P<0.05$ to control (no material), ${ }^{* *}: P<0.01$ to control (no material).

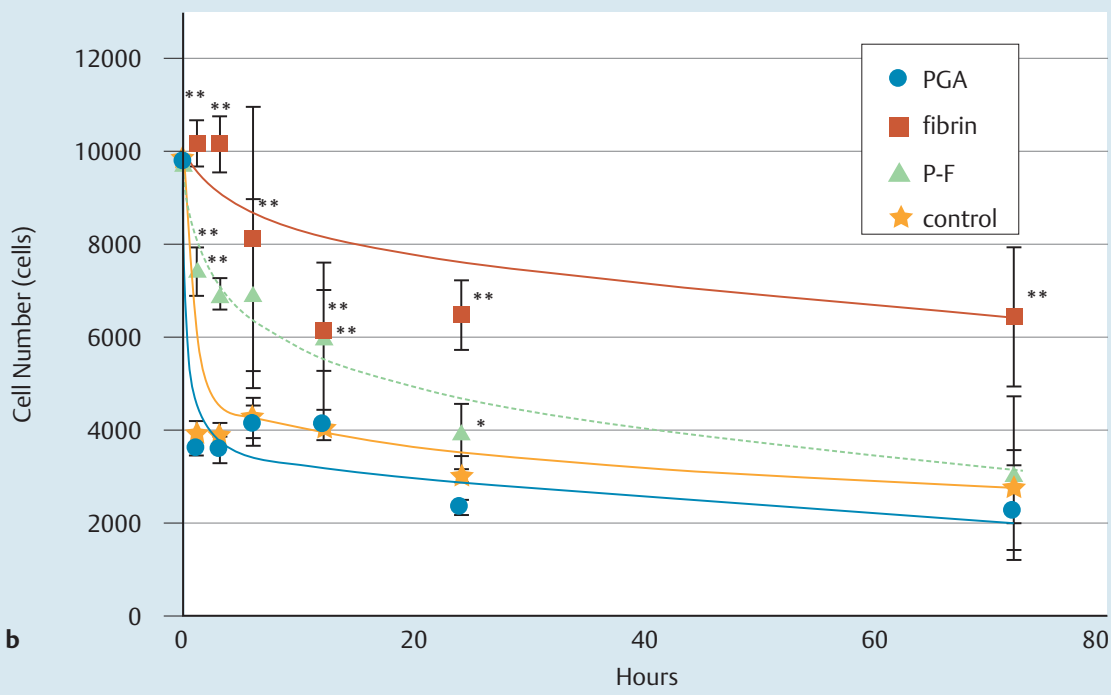

of each material on fibroblasts cultured under hydrochloric acid treatment, which showed that more fibroblasts survived on fibrin followed by a complex of both, and PGA. This result suggests that fibrin glue with or without PGA can exhibit a protective effect against gastric juice, which contains hydrochloric acid. Because the fibrin clot disappeared after up to 3 days in P-F group of our canine ESD model, the protective effect of fibrin glue may mainly protect the surface of the ulcer lesion during the earlier phase, inducing thicker granulation tissues and less tissue damage from gastric juice. After that phase, formation of denser granulation tissues induced partially by PGA fabrics as scaffold may protect the surface of an ulcer lesion and result in excellent tissue repair and mucosal regeneration at a latter phase.

\section{Conclusion}

$\nabla$

The results of this study based on the basic experiments suggest that the P-F method exhibited a protective effect against gastric juice by fibrin glue to reduce tissue damage and the PGA fabric serves a scaffold function, to induce better granulation formation at the earlier phase, resulting in excellent long-term tissue repair of ulcer lesion following ESD.

\section{Competing interests: None}

\section{Institutions}

${ }^{1}$ Division of Medical Life System, Department of Medical Life System, Doshisha University, Kyotanabe, Kyoto, Japan

2 Department of Gastroenterology, Takeda Central Hospital, Kyoto, Japan

${ }^{3}$ Department of Gastroenterology, Kyoto Prefectural University of Medicine, Kyoto, Japan

${ }^{4}$ QOL Institute, Gunze Ltd., Ayabe, Kyoto, Japan

\section{Acknowledgements}

$\nabla$

We appreciate Ms. Akamatsu in CSL-Behring Japan for providing experimental materials. 


\section{References}

1 Gotoda T, Yamamoto H, Soetikno RM et al. Endoscopic submucosal dissection of early gastric cancer. J Gastroenterol 2006; 41: 929-942

2 Oka S, Tanaka S, Kaneko I et al. Advantage of endoscopic submucosal dissection compared with EMR for early gastric cancer. Gastrointest Endosc 2006; 64: 87

3 Saito Y, Uraoka T, Yamaguchi Yet al. A prospective, multicenter study of 1111 colorectal endoscopic submucosal dissections (with video). Gastrointest Endosc 2010; 72: 1217-1225

4 Saito Y, Fukuzawa M, Matsuda T et al. Clinical outcome of endoscopic submucosal dissection versus endoscopic mucosal resection of large colorectal tumors as determined by curative resection. Surg Endsc 2010; $24: 343-352$

5 Otake Y, Saito Y, Sakamoto $T$ et al. New closure technique for large mucosal defects after endoscopic submucosal dissection of colorectal tumors (with video). Gastrointest Endosc 2011; 75: 663-667

6 Takimoto K, Toyonaga T, Matsuyama K. Endoscopic tissue shielding to prevent delayed perforation associated with endoscopic submucosal dissection for duodenal neoplasms. Endoscopy 2012; 44: E414-E415

7 Tsuji Y, Ohata K, Gunji T et al. Endoscopic tissue shielding method with polyglycolic acid sheets and fibrin glue to cover wounds after colorec- tal endoscopic submucosal dissection (with video). Gastrointest Endosc 2014; 79: 151 - 155

8 Takimoto K, Imai Y, Matsuyama K. Endoscopic tissue shielding method with polyglycolic acid sheets and fibrin glue to prevent delayed perforation after duodenal endoscopic submucosal dissection. Dig Endosc 2014; $26: 46-49$

9 Takao T, Takegawa $Y$, Shiya $N$ et al. Tissue shielding with polyglycolic acid sheets and fibrin glue on ulcers induced by endoscopic submucosal dissection in a porcine model. Endos Int Open 2015: E146

10 Ueda $K$, Tanaka $T$, Jinbo $M$ et al. Sutureless pneumatosis using polyglycolic acid mesh as artificial pleura during video-assisted major pulmonary resection. Ann Thorac Surg 2007; 84: 1858 -1861

11 Hayashibe A, Sakamoto K, Shinbo M et al. New method for prevention of bile leakage after hepatic resection. J Surg Oncol 2006; 94: 57-60

12 Uemura K, Murakami Y, Hayashidani Yet al. Combination of polyglycolic acid felt and fibrin glue for prevention of pancreatic fistula following pancreaticoduodenectomy. Hepatogastroenterology 2009; 56: $1538-1541$

13 Shinya N, Oka S, Miyabashira S et al. Improvement of the tissue-adhesive and sealing effect of fibrin sealant using polyglycolic acid felt. J Invest Surg 2009; 22: $383-389$ 